\title{
A SEMIEMPIRICAL METHOD FOR CALCULATING LCAO-MO MATRIX ELEMENTS OF TRANSITION METAL COMPLEXES
}

\author{
R. G. SHULMAN \\ Bell Telephone Laboratories, Incorporated \\ Murray Hill, New Jersey \\ (Received 8 December 1965)
}

IN $\mathrm{KNiF}_{3}$ the cubic crystal field splitting and the spin density transferred to the fluorines have been calculated [1] numerically with an effective one-electron Hamiltonian in an LCAO-MO model and found to be in good agreement with experiment. Extensions of these calculations with a more rigorously derived Hamiltonian [2,3] either have lost this agreement because of the sensitivity of the three-electron calculation to the choice of nickel ion basis functions [2] (i.e. $\mathrm{Ni}^{+}$or $\mathrm{Ni}^{++}$), or else have resorted to a somewhat arbitrary introduction of experimental parameters [3]. A theoretical analysis has shown [4] how the one-electron model avoids this sensitivity and has suggested that correlation effects are responsible for its success. Theoretically, therefore, the situation is quite similar to that which prevailed in $p \pi$ electron calculations of organic molecules after the Goeppert-Mayer and Sklar [5] calculation did not agree very well with the experiments, and the reasons are similar, i.e. the difficulties of evaluating the one center coulomb integral with a limited basis set. Pariser-Parr [6] and Pople [7] eased these difficulties by showing how empirical information could be systematically introduced into the calculations.

I should like to propose a related method for utilizing empirical parameters in calculations of LCAO-MO matrix elements of transition metal complexes. Values calculated on this basis agree very well with those calculated more accurately on the one electron model in $\mathrm{KNiF}_{3}$ and also agree with experimental results in other octahedral complexes. Although the calculated values chosen as examples in this report are confined to the Hamiltonian and matrix elements used in reference [1], the approximations suggested can certainly be used to evaluate the matrix elements obtained from: different starting assumptions.

Because of the experimental insensitivity to non-nearest neighbors discussed previously [1] all calculations will be confined to a cluster of the form $\left(\mathrm{NiF}_{6}\right)^{-4}$ assumed to lie in a constant potential. The assumption of zero differential overlap will be made for the diagonal matrix elements of the Hamiltonian $h_{i i}$ and $h_{j j}$. This becomes equivalent to considering only coulomb integrals between two centers, which for the almost ionic transition metal complexes rather accurately reduce to point charge terms. (Assuming that point charges accurately represent the coulomb contributions to $h_{i}$ is not equivalent to assuming that point charges can be used to calculate the cubic crystal field splitting $\Delta_{\text {. }}$ )

The unpaired electron is in an antibonding LCAO-MO of the form

$$
\psi=N-1 / 2\left\lfloor\phi_{i}-(\gamma+S) \chi_{j}\right]
$$

where $\varphi_{i}$ is the metal function, $x_{j}$ the suitable combination of ligand's wave functions, $S$ the 
metal-ligand overlap and $\gamma$ the covalency parameter. It can be shown that

$$
h_{i i}=\left(\phi_{i}\left|-\frac{\nabla^{2}}{2}+V_{m}\right| \phi_{i}\right)+\left(\varphi_{i}\left|V_{L}\right| \phi_{i}\right) \text {. }
$$

reduces to

$$
h_{i i}=\varepsilon_{d}+\frac{6}{\rho}
$$

where the Hamiltonian

$$
h=-\frac{\nabla 2}{2}+V_{m}+V_{L}
$$

is discussed previously [1]. $V_{m}$ and $V_{L}$ are the metal and ligand core potentials and $-\nabla^{2} / 2$ is the kinetic energy operator. $\varepsilon_{d}$ is the orbital energy of the $3 d$ electron of the metal and $\rho$ is the metal-ligand spacing.

In a similar fashion the diagonal term for the fluoride ligand $p_{\sigma}$ orbitals becomes

$$
h_{j j}=\varepsilon_{\sigma}+\frac{0.329}{P}
$$

The values calculated for $h_{i}$ and $h_{j j}$ from these expressions for $\mathrm{KNiF}_{3}$ are compared in the table with the values calculated by evaluating all two-center integrals numerically. Both approximate values are higher than the more exact by about one electron volt so that the values calculated for the energy difference $h_{i}-h_{j j}$ by the two methods are similar.

The off diagonal matrix element $h_{i j}$ is usually estimated [8]. Calculations of $h_{i j}$ show it to be very sensitive to three center integrals which are difficult to calculate accurately. Furthermore in transition metal complexes, in contrast to aromatic molecules, the same $h_{i j}$ can be used only in one complex. Hence one cannot arrive at estimated values of $h_{i j}$ which can be consistently used in different molecules. In $\mathrm{KNiF}_{3}$ it was shown [1] that $h_{i j}$ made the dominant contribution to the cubic crystal field splitting $\Delta$, thereby justifying a proposal to this effect made by Anderson [9]. Here the argument will be reversed and the value of $\Delta$ in octahedral complexes used to evaluate $h_{i j}$.

From examination of the different contributions to $\Delta$ calculated in $\mathrm{KNiF}_{3}$ one can show that to within reasonable accuracy the $d-p_{\sigma}$ off diagonal matrix element is given by

$$
-\gamma h_{i j}=\Delta-\Delta p c
$$

where $\Delta_{p c}$ is the point charge contribution to $\Delta$ (which is usually a small term) and

$$
\gamma=\frac{h_{i j}-S h_{j j}}{h_{i i}-h_{j j}}
$$

(Four terms which are ignored because they approximately cancelled in the $\mathrm{KNiF}_{3}$ calculation are the nickel diagonal; the off diagonal terms arising from nonorthogonality; the $2 s$ electron contributions; and the contributions from the $p_{\pi}$ electrons.)

If $\gamma$ is not known experimentally it can be expressed as a function of $h_{i j}$ by evaluating the 
diagonal matrix elements as described above. If $\gamma$ is experimentally determined then $h_{i j}$ can be derived from the two observables $\gamma$ and $\Delta$. In $\mathrm{KNiF}_{3}$, for example, the values were

$$
\begin{aligned}
& \gamma_{\sigma}=0.23 \\
& \Delta=7250 \mathrm{~cm}^{-1} \\
& \Delta_{p c}=1390 \mathrm{~cm}^{-1} .
\end{aligned}
$$

This value of $h_{i j}$ calculated from these quantities is given in the table along with the value calculated more precisely. If the approximate values of the diagonal matrix elements had been used to evaluate $\gamma$ the derived value of $h_{i j}$ would not differ significantly from the value listed in Table 1.

TABLE 1

Comparison of matrix elements for $\mathrm{KNiF}_{3}(\mathrm{eV})$

\begin{tabular}{l|c|c|c}
\hline & $h_{i i}$ & $h_{j j}$ & $h_{i j}$ \\
\hline Present approximation & 4.6 & -2.6 & -2.7 \\
\hline Previous calculation & 3.23 & -4.43 & -3.15
\end{tabular}

To extend these results to the $s$ and $p_{\pi}$ ligand orbitals, we suggest that

$$
\begin{aligned}
& \frac{h_{i \sigma}}{h_{i \pi}}=\frac{S_{\sigma}}{S_{\pi}} \\
& h_{i \sigma}=h_{i s} \\
& \delta_{s}=\delta+\varepsilon_{\sigma}-\varepsilon_{s}, \\
& \delta_{\pi}=\delta
\end{aligned}
$$

where

$$
\delta \equiv h_{i i}-h_{j j}
$$

These relations can be confirmed to be valid for $\mathrm{KNiF}_{3}$ and in their assumed dependence upon the overlap follow in part the approximation procedure most widely used [8]. We would like to emphasize that the Wolfsberg-Helmholz approximation for determining $h_{i \sigma}$ must be applied to octahedral complexes with caution because it ignores the three center integrals which are very large. In fact, in $\mathrm{KNiF}_{3}$, they are equal to the values of $h_{i j}$. However, Pople has pointed [10] out that after assuming zero differential overlap it is more consistent, and probably more accurate, to neglect three center integrals than to include them. The same can be inferred from Phillips' [11] 
discussion of transition metal complexes which assumed (as was subsequently confirmed [1]) that in the absence of covalency the simple point charge model was a good approximation to the complete interaction between two ions. From symmetry considerations there are no covalency interactions between completely filled ligand orbitals and the point charge contributions to $h_{i j}$ are included explicitly. In any event the approximations suggested here are more directly based upon experiment and can correlate different observables.

One can test these proposals in $\left(\mathrm{IrCl}_{6}\right)^{2}$ - where the $\pi$ bonding was measured in ESR experiments by Griffiths, Owen and Ward [12] who showed that $f_{\pi} \sim 0.05$. From the measured values of [13] $\Delta=29,000 \mathrm{~cm}^{-1}, \rho=4.3$ a.u., $q=1$ and $n=4$ and the assumed values of $\varepsilon_{d} \approx-45 \mathrm{eV}$ (a rough estimate of the orbital energy of the $d$ electrons in $\mathrm{Ir}^{+4}$ ) and $\varepsilon_{p} \approx-5 \mathrm{eV}$ we calculate that $\delta \approx 8 \mathrm{eV}$ and $h_{i \sigma} \approx 5.5 \mathrm{eV}$. This leads to a value of $\gamma_{\sigma} \approx 0.6$ and $f_{\sigma}=\left(\gamma_{\sigma}+S_{\sigma}\right)^{2 / 3} \approx 0.15$. From the use of equation (14) one obtains $f_{\pi} \approx 0.05$ which agrees with the observed [12] value. This agreement is only approximate because of the need to assume a value of $\varepsilon_{d}$. However, it is not a sensitive function of this assumption because larger values of $\delta$ will not change $\gamma_{\sigma}$ significantly while smaller values would lead to unrealistically low energies for charge transfer.

Although this proposed method of evaluating matrix elements is approximate, it is capable of being applied to the $\mathrm{IrCl}_{6}{ }^{-2}$ complex, which is quite dissimilar to the $\mathrm{NiF}_{6}{ }^{4-}$ complex upon which the approximations are based, and should have greater physical validity than less empirical, approximate methods.

\section{References}

1. S. Sugano and R.g. Shulman, Phys. Rev. 130, 517 (1963).

2. E. SIMANEK and Z. SROUBEK, Phys. Stat. Solidi 4, 251 (1964).

3. R.E. WATSON and A.J. FREEMAN, Phys, Rev. 134, A1526 (1964).

4. S. SUgANO and Y. TANABE, J. Phys, Soc., Japan 20, 1155 (1965).

5. M. GOePPert-mayer and A.L. Sklar, J. Chem. Phys. 6, 645 (1938).

6. R.G. PARR and R. PARISER, J. Chem. Phys, 23, 711 (1955).

7. J.A. POPLE, Trans. Faraday Soc. 49, 1375 (1953).

8. M. WOLfSberg and L. helmholz, J. Chem. Phys, 20, 837 (1952).

9. P.W. ANDERSON, Phys. Rev. 115, 2 (1959).

10. J.A. POPLE, private communication.

11. J.C. PHIllips, J. Phys. Chem. Solids 11, 226 (1959).

12. J.H.E. GRIFFith, J. OWEN and I.M. WARD, Proc. Roy. Soc., Lond. A219, 526 (1953).

13. C.K. JORGENSEN, Absorption Spectra and Chemical Bonding in Complexes, p. 155. Pergamon Press (1962). 